\title{
Erratum to: Spectral Gap and Logarithmic Sobolev Constant for Continuous Spin Systems
}

\author{
Mu Fa CHEN \\ School of Mathematical Sciences, Beijing Normal University, \\ Beijing 100875, P. R. China \\ E-mail:mfchen@bnu.edu.cn
}

\section{Erratum to: Acta Mathematica Sinica, English Series}

DOI: $10.1007 /$ s10114-007-7293-3

Two figures were unfortunately missed in the publication: Figure 1 should be below Theorem 6.3 and Figure 2 should be above Remark 6.4.

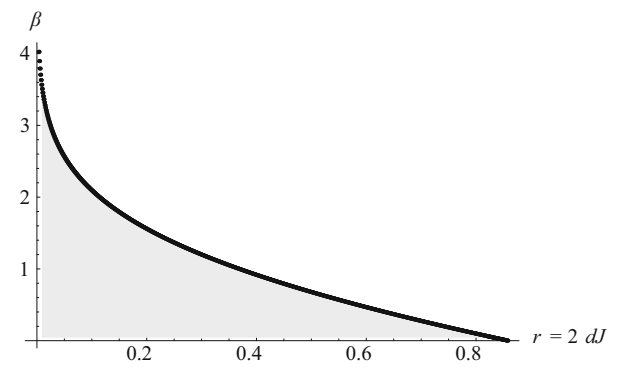

Figure 1

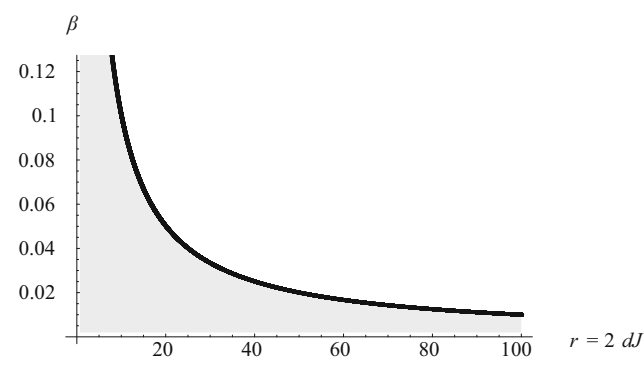

Figure 2

The online version of the original article can be found at http://dx.doi.org/10.1007/s10114-007-7293-3 